\title{
CARDIO-RESPIRATORY CHANGES AND MORTALITY IN THE CONSCIOUS RAT INDUCED BY (+)- AND ( \pm -ANATOXIN-a
}

\author{
Oluwadare M. Adeyemo and AnNa-Leena Sirén \\ Department of Neurology, Uniformed Services University of the Health Sciences, 4301 Jones Bridge Road, \\ Bethesda, MD 20889-4799, U.S.A.
}

(Received 18 November 1991; accepted 13 February 1992)

\begin{abstract}
O. M. AdEYEmo and A.-L. SrRÉn. Cardio-respiratory changes and mortality in the conscious rat induced by $(t)$ - and $( \pm)$-anatoxin-a. Toxicon 30, 899-905, 1992.-Anatoxin-a (AnTx-a) is a potent nicotinic cholinergic receptor agonist. The relative potencies of the $(+)$-AnTx-a and the racemic mixture $( \pm)$-AnTxa were investigated in the conscious rat by comparing their effects on mean arterial blood pressure (BP), heart rate (HR), blood oxygen and carbon dioxide pressures $\left(\mathrm{pO}_{2}\right.$ and $p \mathrm{CO}_{2}$, respectively), acid-base balance $(\mathrm{pH})$ and mortality. The present experiments show that while both forms of AnTx-a produce dose-dependent increases in BP and decreases in HR, (+)-AnTx-a is about 10-fold more potent than the optically inactive isomer. ( + -AnTx-a was also 6-fold more potent than ( \pm )-AnTx-a in producing severe hypoxemia, and more than 4-fold as potent as the $( \pm)$-AnTx-a in producing significant hypercapnia accompanied with severe acidosis. The approximate median lethal dose $\left(\mathrm{LD}_{s_{0}}\right)$ of $(+)$-AnTx-a was about 5 -fold less than that of $( \pm)$-AnTx-a. We conclude that $(+)$-AnTx-a is more potent than the $( \pm)$-AnTx-a racemic mixture in causing detrimental cardio-respiratory changes and therefore increased mortality in the rat.
\end{abstract}

\section{INTRODUCTION}

ANATOXIN-a (AnTx-a) is a naturally occurring semirigid alkaloid neurotoxin with a bicyclic secondary amine structure, isolated from the blue-green algae Anabaena flosaquae (CARMICHAEL, et al., 1975; KOSKINEN and RAPOPORT, 1985). This neurotoxin was shown to be a potent stereospecific agonist at nicotinic synapses in torpedo electric organs and frog muscle (CARmachael et al., 1979; Aronstam and WrTkop, 1981; AlbuQUERQUe and SPIVAK, 1984; Swanson et al., 1986; ArAcava et al., 1988; AlbuQUERQUE et al., 1989).

In vitro studies have shown that $(+)$-AnTx-a was more than twice as potent as the racemic mixture $( \pm)$-AnTx-a on nicotinic receptor mediated contractile response of rectus abdominis muscle of the frog Rana-pipiens (SPIVAK et al., 1980, 1983). The (+)-AnTx-a was also more than 50-fold more potent than ( \pm -AnTx-a in inhibiting the specific binding of $\left[{ }^{125} 1\right]$ - $\alpha$-bungarotoxin to the nicotinic receptors in torpedo electric organ (Swanson $e t$ al., 1986). In the rat brain, (+)-AnTx-a binds to nicotinic receptors with a 20-fold higher potency than ( \pm )-AnTx-a and was more than 160 -fold as potent as (-)AnTx-a in inhibiting $\left[{ }^{3} \mathrm{H}\right]$-acetylcholine binding to the rat's cortex, suggesting that the 
enantiomer (-)-AnTx-a in the racemic mixture is a weak agonist (Swanson et al., 1986; ZhaNG et al., 1987; Koful et al., 1990).

The existence of different subtypes of nicotinic receptors in the rodent brain has been described by LARSSON and NORDBERG (1985). In recent studies, the affinities of $(+)$ - and $( \pm)$-AnTx-a for the nicotinic receptors were shown to be significantly different. $(+)$ AnTx-a binds to high affinity sites $\left(K_{d}=6 \mathrm{nM}\right)$ while $( \pm)$-AnTx-a binds to low affinity sites $\left(K_{d}=125 \mathrm{nM}\right)$ of nicotinic receptor in the rat's cortex (ZHANG et al., 1987; NORDBERG et al., 1988; KoFuJI et al., 1990). The present studies were undertaken to investigate in vivo the role of enantiomorphism of the AnTx-a molecule. Thus, mean arterial pressure, heart rate, respiratory gases, and mortality were monitored after systemic administration of $(+)$ - or $( \pm)$-AnTx-a in the conscious rat.

\section{MATERIALS AND METHODS}

Male Sprague-Dawley rats (Taconic Farms, Germantown, NY, U.S.A.) weighing $310 \pm 5 \mathrm{~g}(n=60)$ were housed in a temperature-controlled room with a $12 / 12 \mathrm{hr}$ light/dark cycle and access to food and water ad libitum.

Measurement of blood pressure, heart rate, blood gases, $\mathrm{pH}$, and mortality

Each rat was anesthetized with halothane ( $2 \%$ in oxygen) and PE-50 catheters were inserted into the right femoral artery and vein. The catheters were tunneled under the skin and exited at the back of the neck. All rats were allowed 3-5 hr to fully recover and stabilize from surgery. The arterial line was connected to a pressure transducer (Narco Bio-Systems model RP 1500) attached to a strain gauge coupler (Narco Bio-Systems Type 7179). Blood pressure and heart rate were recorded on a Narcotrace 80 computerized physiograph and sampled manually as required via a Northstar Hazeltine computer.

All drugs and saline were administered through the i.v. line in a total volume of $0.2 \mathrm{ml}$ which was immediately followed with a $0.3 \mathrm{ml}$ saline wash. The response to drug administration was obvious immediately after injection with peak effect within 1-2 min, which completely subsided in about $15 \mathrm{~min}$. Samples for all parameters except mortality data were taken within this peak effect period.

For the blood gas measurements, a sample $(0.4 \mathrm{ml})$ of heparinized arterial blood was taken via the arterial catheter immediately before anatoxin-a or saline treatments and at the peak of the cardiovascular response. Equal volumes of saline were used for the replacement of blood withdrawn. These samples were immediately analyzed for $\mathrm{pO}_{2}, \mathrm{pCO}_{2}$, and $\mathrm{pH}$ values, using a STAT Profile 3 blood gas analyzer (Nova Biomedical, Waltham, MA, U.S.A.). Mortality data were monitored at all AnTx-a dose levels within an observation period of $30 \mathrm{~min}$ because if the dose was not fatal, total recovery from AnTx-a effects usually occurred about 15 min after the administration of AnTx-a. All surviving rats were monitored for $2 \mathrm{hr}$ and finally sacrificed with T-61 euthanasia solution containing embutramide, mebezonium iodide, and tectracaine hydrochloride.

\section{Drugs used}

(+)-AnTx-a was purchased from Bio-Metric Systems, Inc. (Eden Prairie, MN, U.S.A.). ( \pm )-AnTx-a was synthesized by Dr RuCK L. DANFriser (MIT) (DANHElser et al., 1985). (+)-and ( \pm )-AnTx-a were dissolved in ethanol separately and the stock solutions were stored at $-20^{\circ} \mathrm{C}$ under argon until used. At the time of use, the drugs were reconstituted in physiological saline (0.9\%) and injected. T-61 euthanasia solution was purchased from Hoechst (Somerville, NJ, U.S.A.).

\section{Statistical analysis of the dato}

Data in figures are mean \pm S.E. for indicated number of rats. Analysis of variance (ANOVA) followed by Dunnett's test was used to analyze data. The approximate median lethal dose $\left(L D_{s 0}\right)$ of $(+)$ and $( \pm)-A n T x-a$ in rat was calculated according to the Spearman-Kärber method (FINNEY, 1978). A significant change was accepted at $\boldsymbol{P}<\mathbf{0 . 0 5}$. 


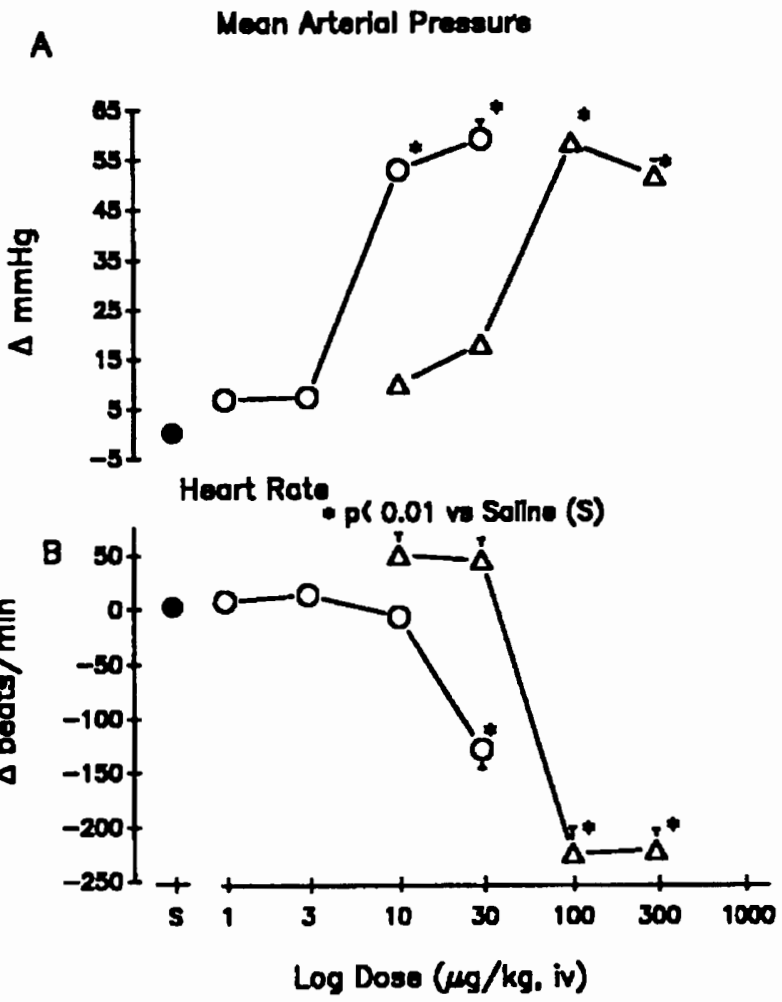

Fig. 1. EFFECT OF i.v. ADMINISTERED (+) AND ( UPPER PANEL) AND HEART RATE (B, LOWER PANEL) IN THE CONSCIOUS RAT.

The values (mean \pm S.E.) indicate maximum changes from baseline. Onset of response was within 5-10 sec, with duration of action of about $15 \mathrm{~min}$ and peak effect within $2 \mathrm{~min}$ following drug treatment. Closed circles denote the effect of saline (S), open circles denote the effects of $(+)$ AnTx-a, and open triangles denote the effect of ( \pm -AnTx-a. Asterisks indicate significant difference from saline control (Dunnett's test). Number of animals is $4-6$ rats per dose.

\section{RESULTS}

Different doses of $(+)$ - and ( \pm )-AnTx-a ranging from 1 to $500 \mu \mathrm{g} / \mathrm{kg}$ were injected i.v. into the rat $\left(n=4-6\right.$ per dose level) and changes in $\mathrm{BP}, \mathrm{HR}, p \mathrm{O}_{2}, p \mathrm{CO}_{2}$, and $\mathrm{pH}$ were monitored. Each animal received a single dose of the drug to prevent any cumulative bias. Both the (+)-AnTx-a and ( \pm -AnTx-a produced simultaneous dose-dependent increases in BP and decreases of more than 100 beats per min in HR. (+)-AnTx-a produced significant changes in BP and HR at lower doses of 10 to $50 \mu \mathrm{g} / \mathrm{kg}$ as compared to the (士)-racemic mixture at relatively higher i.v. doses of 100 to $300 \mu \mathrm{g} / \mathrm{kg}$ (Fig. 1). Potency comparisons between the two forms of AnTx-a showed that the (+)-form was about 10fold more potent than the \pm -isomer.

(+)-AnTx-a induced significant hypoxemia in the rat at a dose of $50 \mu \mathrm{g} / \mathrm{kg}$, i.v., while a significant decrease in the arterial $\mathrm{pO}_{2}$ was not observed with $( \pm)$-AnTx-a until the relatively high dose of $300 \mu \mathrm{g} / \mathrm{kg}$, i.v. (Fig. 2A). Similar responses were observed with changes in $\mathrm{pCO}_{2}$ values. Significant hypercapnia was evident with $(+)$-AnTx-a treatment at $70 \mu \mathrm{g} / \mathrm{kg}$, i.v., as compared to $300 \mu \mathrm{g} / \mathrm{kg}$, i.v. for ( \pm )-AnTx-a (Fig. 2B). (+)-AnTx-a treatment caused hypercapnia accompanied by severe acidosis $(P<0.05)$, while $( \pm)$ AnTx-a even at higher doses failed to produce any significant change in arterial blood acid-base balance (Fig. 2C). 


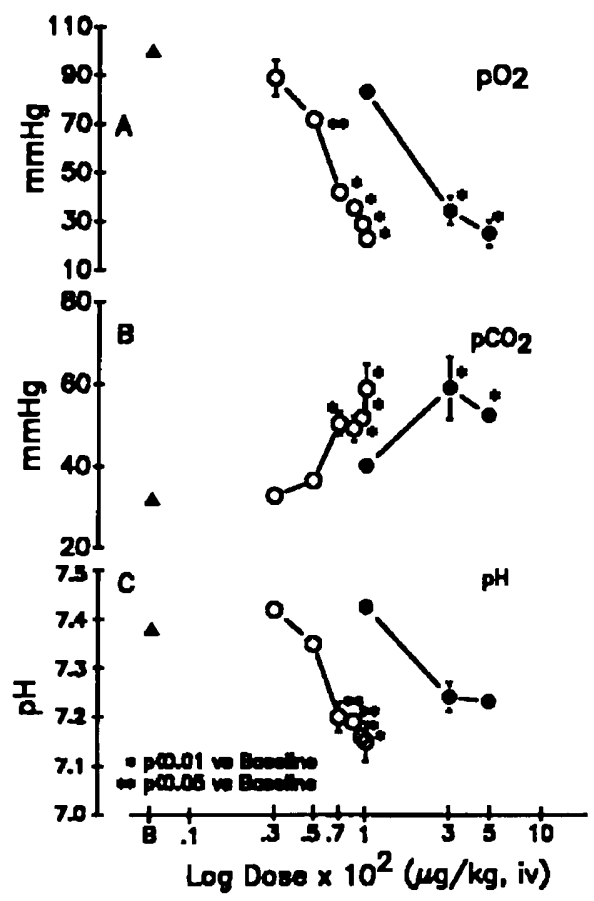

Fig. 2. EFFECT OF i.v. ADMINISTERED ( + )- AND ( \pm )-AnTX-a ON BLOOD GASES: $p_{2}$ (A, UPPER-PANEL), $\mathrm{pCO}_{2}$ (B, middle PANEL), AND ACID- base BalaNCE (pH) (C, LOWER PANEL) IN THE CONSCIOUS RAT. The values are mean \pm S.E. Closed triangles denote the baseline (B), open circles denote the effect of $(+)-A n T x-a$, and closed circles denote the effect of $( \pm)-A n T x-a$. Significant differences from baseline are indicated by asterisks (Dunnett's). Number of animals is $4-6$ rats per dose.

The time-courses of the autonomic effects of both the $(+)$-isomer and the $( \pm)$-isomer were identical. The changes in cardiovascular and respiratory gases became apparent 5-10 sec after AnTx-a administration and subsided within 5-15 min. The peak effect was reached at about $2 \mathrm{~min}$ after i.v. injections. Usually, all rats surviving for more than $30 \mathrm{~min}$ after AnTx-a treatments survived indefinitely. Therefore, the duration for the approximate $\mathrm{LD}_{50}$ calculations was $30 \mathrm{~min}$. The i.v. $\mathrm{LD}_{30}$ dose in rats for ( + )-AnTx-a was $\simeq 85 \mu \mathrm{g} / \mathrm{kg}$, and for ( \pm )-AnTx-a was $\simeq 400 \mu \mathrm{g} / \mathrm{kg}$ (Fig. 3).

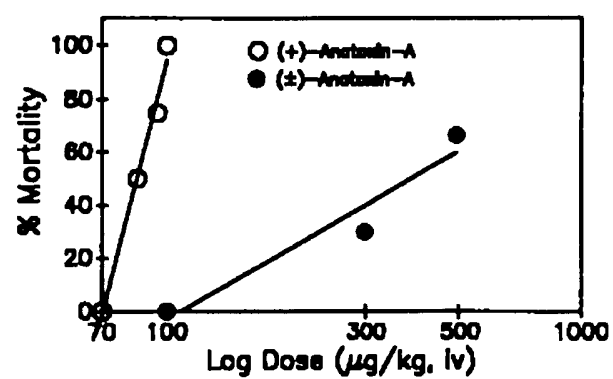

Fuo. 3. THE LETHAL EFFECT Of i.v. ADMINISTERED ( + )- AND ( \pm )-AnTx-a IN THE CONBCTOUS RAT. The approximate median lethal dose $\left(L D_{s 0}\right)$ within a $30-\min$ period of observation is calculated using the Spearman-Käber method. The i.v. $L^{20}$ for $(+)-A n T x-a$ is $\simeq 85 \mu \mathrm{g} / \mathrm{kg}$, and for $( \pm)-A n T x-a$ is $\simeq 400 \mu \mathrm{g} / \mathrm{kg}$. Open circles denote the lethal effect of $(+)$-AnTx-a and closed circles represent the lethal effect of $( \pm)$-AnTx-a in rat. Number of animals is 4-6 rats per dose. 
DISCUSSION

In the present study, (+)-AnTx-a was more potent than ( $($ )-AnTx-a in producing severe disturbances in cardiovascular parameters and respiratory gases. (+)-AnTx-a produced significant changes in $\mathrm{BP}, \mathrm{HR}, \mathrm{pO}_{2}, \mathrm{pCO}_{2}$, and $\mathrm{pH}$ at lower i.v. doses than the $( \pm)$-racemate. Furthermore, the approximate $\mathrm{LD}_{50}$ dose for the $(+)$-AnTx-a was about 5-fold less than that of $( \pm)$-AnTx-a. The data and visual observations from the present experiment suggest that drug-induced mortality is associated with severe changes in blood gases (primarily hypoxia) brought about by a combination of direct cardiovascular effects, and respiratory paralysis caused by AnTx-a.

The difference in potencies between $(+)$ - and $( \pm)-A n T x-a$, as shown by changes in BP and HR, is most likely associated with their binding affinities for and stimulation of the nicotinic cholinergic receptors. Previous in vitro studies have shown that the $(+),(-)$, and $( \pm)$ isomers of AnTx-a bind to the nicotinic receptors with varying affinities (SwANSON $e t$ al., 1986; ZhANG et al., 1987). When the relative potencies ( $\left.\mathrm{IC}_{\text {S0 }}\right)$ of AnTx-a isomers and nicotine were compared, $(+)$-AnTx-a was more than 20 -fold more potent than $( \pm)$ AnTx-a and twice as potent than nicotine in inhibiting $\left[{ }^{3} \mathrm{H}\right]$-acetylcholine specific binding at the receptor site in the rat brain (ZHANG et al., 1987). Also in recent studies from our laboratory, ( \pm -AnTx-a was more than 10-fold less potent than nicotine (SIREN and FeUERsteIn, 1990). Thus the reduced potency of the $( \pm)$-AnTx-a may be due to its binding to the low affinity site which is not known to mediate any physiologic responses.

In explaining a probable mechanism for AnTx-a toxicosis, based upon the present data, a similar nicotine-associated hemodynamic disturbance could be considered. Nicotine is known to exert its effects at both peripheral and central sites in the nervous system. It has also been shown to produce increased blood pressure associated with stimulation of the sympathetic ganglia, adrenal medulla, carotid and aortic chemoreceptor, and direct vasomotor stimulation (COMROE, 1960; ARMTTAGE, 1965). Similarly to nicotine, AnTx-a-induced hemodynamic changes were believed to be associated with its effects on multiple sites in the nervous system. For example, SIRÉN and FeuERSTEIN (1990) reported that the increased blood pressure observed in the rat following AnTx-a administration was caused by increases in cardiac output and vasoconstriction in the renal and mesenteric circulation. Furthermore, the accompanying bradycardia was associated with reflex vagal activation since the vasoconstrictive response came first. These effects were believed to be mediated by nicotinic receptors, as evidenced by the complete blockade of these AnTx-a effects by chlorisondamine.

Blood gases are among the most fundamental of all tests of pulmonary performance. The occurrence of hypoxia in concert with hypercapnia suggest severe hypoventilation and circulatory insufficiency. The severe hypoxia observed in the present experiment may be brought about by the direct effect of AnTx-a on the respiratory muscles and the diaphragm, resulting in paralysis. This conclusion is supported by studies of CARMICHAEL $e t$ al. (1975) and ValentnNe et al. (1991). Generally, hypoxia is associated with interactions between reflex, nerve-mediated responses, and local influence of blood gas tension changes, e.g. reflex effects of peripheral chemoreceptors stimulation by hypoxia or direct effects of $\mathrm{pCO}_{2}$ and $\mathrm{pO}_{2}$ (DALY, 1986). The occurrence of bradycardia in the presence of vasoconstriction in most vascular beds, a phenomenon attributable to sustained stimulation of peripheral carotid bodies with hypoxic blood, has been reported in other studies (DALY, 1983, 1986). 
Since adequate delivery of oxygen to the brain is of prime importance for proper CNS function, the observation that AnTx-a-induced hypoxia was accompanied by severe and sometimes fatal acidosis suggests that brain hypoxia at the cellular level may result in the accumulation of lactate via anaerobic glycolysis producing acid-base stress, probable loss of reducing equivalents, and rapid depletion of high-energy phosphate compounds produced through oxidative phosphorylation. HrTzIG (1989) reported that brain cells are resistant to moderate hypoxia and can compensate for acid-base imbalance associated with hypoxia; but under severe hypoxia, brain cells lose their compensatory ability. This may explain why not all the animals treated with median doses of the more potent $(t)-$ AnTx-a survived and relatively large doses of the less potent $( \pm)$-AnTx-a were not fatal in some animals. We therefore conclude from our data that the depression of the brain respiratory center brought about by pronounced hypoxia, followed by respiratory acidosis, and compounded by respiratory muscle paralysis are contributing factors to the expression of toxicity to AnTx-a. This study provides direct evidence for additional mechanisms of toxicity by AnTx-a.

Acknowledgement-This work was supported by the United States Army Medical Research and Development Command Protocol \# G49231. The opinions and assertions contained herein are the private ones of the authors and are not to be construed as official or as necessarily reflecting the views of the Department of Defense or the Uniformed Services University of the Health Sciences. The experiments reported herein were conducted according to the principles set forth in the Guide for Care and Use of Laboratory Animals Resources, National Research Council (DHEW Publication No. NIH 85-23, 1985).

\section{REFERENCES}

Albuquerque, E. X. and Sprvar, C. E. (1984) Natural toxins and their analogues that activate and block the ionic channel of the nicotinic acetylcholine receptor. In: Natural Products and Drug Development, Alfred Benzon Symposium, Vol. 20, pp. 301-321 (Krogsgand-Larsen, P., Christensen, S. B. and Kofod, H., Eds). Copenhagen: Munksgard.

Albuquerque, E. X., Alrondon, M., lima-landman, M. T., Deshpande, S. S. and Ramoa, A. S. (1989) Molecular targets of noncompetitive blockers at the central and peripheral nicotinic and glutamatergic receptors. In: Neuromuscular Junction, Fernstrom Foundation Series, Vol. 13, pp. 273-300 (SeluINs, L. S., Libelins, R. and TheslefT, S., Eds). Cambridge: Elsevier.

Aracava, Y., Swanson, K. L., Rozental, R. and Albuquerque, E. X. (1988) Structure-activity relationship of ( +)-anatoxin-a derivatives and enantiomers of nicotine on the peripheral and central nicotinic acetylcholine receptor subtypes. In: Neurotoxicology, Vol. 88, Molecular Basis of Drug and Pesticide Action, pp. 157-184 (LuNT, G. G., Ed.). Cambridge: Elsevier.

ARMITAGE, A. K. (1965) Effect of nicotine and tobacco smoke on blood pressure and release of catecholamine from the adrenal gland. Br. J. Pharmac. 25, 515-526.

AronstaM, R. S. and WrTxop, B. (1981) Anatoxin-a interactions with cholinergic synaptic molecules. Proc. natn. Acad. Sci. U.S.A. 78, 4639-4643.

Carmichael, W. W., Bigos, D. F. and Gorham, P. R. (1975) Toxicology and pharmacological action of Anabaena flos-aquae toxin. Science 187, 542-544.

Carmichael, W. W., Biggs, D. F. and Peterson, M. A. (1979) Pharmacology of anatoxin-a, produced by fresh water cynophyte Anabaena flos-aquae NRC-44-1. Toxicon 17, 229-236.

Comroe, J. H., JR (1960) The pharmacological actions of nicotine. Ann. N.Y. Acad. Sci. 90, 48-51.

DALY, M. DE B. (1983) Peripheral arterial chemoreceptors and the cardiovascular system. In: Physiology of Peripheral Arterial Chemoreceptors, pp. 325-393 (ACKER, H. and O'REGAN, R. G., Eds). Amsterdam: Elsevier.

DALY, M. DE B. (1986) Interactions between respiration and circulation. In: Handbook of Physiology-the Respiratory System, II, Section 3, Part 2, pp. 529-594 (Chrerniacx, N. S. and Widdicombe, J. G., Eds). Bethosda: American Physiological Society.

Danfeiser, R. L., Morin, J. M. and Salasku, E. J. (1985) Efficient total synthesis of (土)-anatoxin-a. J. Am. Chem. Soc. 107, 8066-8073.

FINNEY, D. J. (1978) Assays based on quantal response; Spearman-Kärber and moving averages. In: Statistical Method in Biological Assays, 3rd Edn, pp. 394-401. London: Griffin \& Co. 
Hrrzig, B. M. (1989) Effect of hypoxia on brain cell acid-base and high energy phosphate regulation by ${ }^{31}$ P-NMR spectroscopy. In: Chemoreceptors and Reflexes in Breathing-Cellular and Molecular Aspects, pp. 255-266 (Lahari, S., Forster, R. E., Davies, R. O. and Park, A. I., Eds). New York: Oxford University Press. Kofun, P., Aracava, Y., Swanson, K. L., Aronstam, R. S., Rapoport, H. and Albuquerque, E. X. (1990) Activation and blockade of the acetylcholine receptor-ion channel by the agonists (+)-anatoxin-a. the $N$-methyl derivative and the enantiomer. J. Pharmac. exp. Ther. 525, 517-525.

KOSKINEN, A. M. P. and RAPOPORT, H. (1985) Synthetic, conformational and pharmacological studies of anatoxin-a, a potent acetylcholine agonist. J. Med. Chem. 28, 1301-1309.

LArsson, C. and Nordberg, A. (1985) Comparative analysis of nicotine-like ligand interactions in rodent brain homogenates. J. Neurochem. 45, 24-32.

Nordberg, A., Adem, A., Nusson, L., Romanelu, L. and Zhang, X. (1988) Heterogenous cholinergic nicotinic receptors in the CNS. In: Nicotinic Acetylcholine Receptors in the Nervous System, NATO ASI series, Vol. H25, pp. 331-350 (Clementi, F. el al., Eds). Berlin Heidelberg: Springer.

Siren A.-L. and Feurrsten, G. (1990) Cardiovascular effects of anatoxin-a in the conscious rat. Toxic. appl. Pharmac. 102, 91-100.

SpIVAK, C. E., Witkop, E. B. and AlbuQUERQUE, E. X. (1980) Anatoxin-a: a novel, potent agonist at the nicotinic receptor. Molec. Pharmac. 18, 384-394.

Spivak, C. E., Waters, J., WiTKop, B. and Albuquerque, E. X. (1983) Potencies and channel properties induced by semirigid agonists at frog nicotinic acetylcholine receptors. Molec. Pharmac. 23, 337-343.

Swanson, K. L., Allen, C. N., Aronstam, R. S., Rapoport, H. and Albuquerque, E. X. (1986) Molecular mechanisms of the potent and stereospecific nicotinic receptor agonist (+)-anatoxin-a. Molec. Pharmac. 29, 250-257.

Valentine, W. M., Sckuefrer, D. J. and Beasley, V. R. (1991) Electromyographic assessment of the neuromuscular blockade produced in vivo by anatoxin-a in the rat. Toxicon 29, 347-357.

Zhang, X., Stjernlö, P., Adem, A. and Nordberg, A. (1987) Anatoxin-a a potent ligand for nicotinic cholinergic receptors in rat brain. Eur. J. Pharmac. 135, 457-458. 